Алгебра и анализ

Том. 17 (2005), вып. 2
St. Petersburg Math. J.

Vol. 17 (2006), No. 2, Pages 217-238

S 1061-0022(06)00901-0

Article electronically published on February 10, 2006

\title{
THOMSON'S THEOREM ON MEAN SQUARE POLYNOMIAL APPROXIMATION
}

\author{
J. E. BRENNAN
}

\begin{abstract}
In 1991, J. E. Thomson determined completely the structure of $H^{2}(\mu)$, the closed subspace of $L^{2}(\mu)$ that is spanned by the polynomials, whenever $\mu$ is a compactly supported measure in the complex plane. As a consequence he was able to show that if $H^{2}(\mu) \neq L^{2}(\mu)$, then every function $f \in H^{2}(\mu)$ admits an analytic extension to a fixed open set $\Omega$, thereby confirming in this context a phenomenon noted earlier in various situations by S. N. Bernštein, S. N. Mergelyan, and others. Here we present a new proof of Thomson's results, based on Tolsa's recent work on the semiadditivity of analytic capacity, which gives more information and is applicable to other problems as well.
\end{abstract}

\section{§1. INTRODUCTION}

Questions concerning approximation by analytic functions have a long history and have arisen in a variety of disparate settings. Of particular interest here is one such problem having implications well beyond the immediate context in which it is phrased. Given a positive measure $\mu$ of compact support in the complex plane $\mathbb{C}$, are the polynomials dense in $L^{2}(d \mu)$; if not, why not? Over the years that question has been the subject of an intense investigation, not only for its own sake, but due also to its connection with the invariant subspace problem for subnormal operators on a Hilbert space.

A bounded linear operator $T$ on an infinite-dimensional Hilbert space $H$ is subnormal if it has a normal extension to a larger Hilbert space; or equivalently, if $T$ is the restriction of a normal operator to a closed invariant subspace. The study of such operators was begun by Halmos [17] in 1950, and since then it has become a catalyst for much activity at the interface between operator theory and the theory of analytic functions.

It is a long standing open problem to determine whether or not every bounded linear operator $T$ on a Hilbert space $H$ has a nontrivial closed invariant subspace. Clearly, it can be assumed from the outset that $T$ has a cyclic vector, that is, a vector $x$ for which the linear span of $x, T x, T^{2} x, \ldots$ is dense in the underlying space $H$. Otherwise, invariant subspaces abound and there is nothing to prove. If, in addition, $T$ is subnormal, the spectral theorem guarantees that there is a positive measure $\mu$ carried on the spectrum of $T$ such that the given operator $T$ is unitarily equivalent to multiplication by the complex identity function $z$ on $H^{2}(d \mu)$. Here $H^{2}(d \mu)$ is the closure of the polynomials in $L^{2}(d \mu)$. Thus, the study of subnormal operators leads readily to questions concerning approximation by polynomials in $L^{2}(d \mu)$ (cf. Bram [3, pp. 83-86]).

In this setting invariant subspaces can arise in at least one of two ways. If $H^{2}(d \mu)=$ $L^{2}(d \mu)$ and if $X$ is any subset of the support of $\mu$ with $0<\mu(X)<\|\mu\|$, then $S=$ $\left\{f \in H^{2}(d \mu): f=0\right.$ a.e.- $d \mu$ on $\left.X\right\}$ is a nontrivial closed subspace invariant under multiplication by $z$. On the other hand, if $H^{2}(d \mu) \neq L^{2}(d \mu)$ it may happen that there

2000 Mathematics Subject Classification. Primary 41A10, 30E10, 31A15, 47B20.

Key words and phrases. Polynomial approximation, analytic capacity, subnormal operators. 
is a point $\xi \in \mathbb{C}$ such that the map $P \rightarrow P(\xi)$ can be extended from the polynomials to a bounded linear functional on $H^{2}(d \mu)$; that is,

$$
|P(\xi)| \leq C\|P\|_{L^{2}(d \mu)}
$$

for every polynomial $P$ and some absolute constant $C$. Such a point $\xi$ is called a bounded point evaluation or BPE for $H^{2}(d \mu)$. If we let $S$ be the closure in $H^{2}(d \mu)$ of the set of polynomials vanishing at $\xi$ we again obtain a nontrivial invariant subspace, since $(z-\xi) \in S$ and $1 \notin S$.

In order, therefore, to settle the invariant subspace problem for subnormal operators in the affirmative it is sufficient to prove that either

(1) $H^{2}(d \mu)$ has a BPE, or

(2) $H^{2}(d \mu)=L^{2}(d \mu)$.

Moreover, initial confirmation that the suggested dichotomy is valid for a large class of measures came quickly. In 1955, Wermer [48] verified it for all measures $\mu$ whose closed support $X$ has two-dimensional Lebesgue measure (i.e., $d A$ measure) zero. His reasoning made use of a theorem of Hartogs and Rosenthal [18 on uniform rational approximation, but in essence is this: Let $g$ be any function in $L^{2}(d \mu)$ that is orthogonal to the polynomials in the sense that $\int P g d \mu=0$ for every polynomial $P$. By an argument due essentially to Cauchy we find that

$$
P(\xi)=\frac{1}{\widehat{g \mu}(\xi)} \int \frac{P(z) g(z)}{z-\xi} d \mu(z)
$$

at every point $\xi \in \mathbb{C}$ where $\widehat{g \mu}(\xi)=\int \frac{g(z)}{z-\xi} d \mu(z)$ is defined and $\widehat{g \mu}(\xi) \neq 0$. In particular, if $\xi \in \mathbb{C} \backslash X$ and $\widehat{g \mu}(\xi) \neq 0$, then (1.2) holds and, since the kernel $(z-\xi)^{-1}$ is bounded on $X$,

$$
|P(\xi)| \leq C \int|P||g| d \mu
$$

for all polynomials $P$ and a suitable constant $C$. Hence, the inequality (1.1) is also satisfied and $H^{2}(d \mu)$ has a BPE at $\xi$. If, therefore, $H^{2}(d \mu)$ has no BPEs, it follows that $\widehat{g \mu}(\xi)=0$ in $\mathbb{C} \backslash X$; that is, $\widehat{g \mu}=0$ a.e.- $d A$ in $\mathbb{C}$, since $X$ has area zero. Thus, $g \mu=0$ as a measure, and so $H^{2}(d \mu)=L^{2}(d \mu)$.

Subsequent efforts to extend this line of reasoning to absolutely continuous measures $w d A$ would yield clues eventually leading to a complete solution of the bounded point evaluation problem. In a very real sense the essential difficulties can all be found here. Building on ideas introduced in [5] in order to validate the BPE alternative for $H^{2}(w d A)$ when $w \in L^{1+\epsilon}(d A)$, Thomson [41] overcame substantial technical difficulties to achieve a final resolution. His result is the following.

Theorem 1. For any measure $\mu$ of compact support, not concentrated at a single point, $H^{2}(d \mu)=L^{2}(d \mu)$ if and only if $H^{2}(d \mu)$ has no BPEs.

Thomson's argument is quite complicated, incorporating an array of deep ideas from Brown [7, Davie [11, and Vitushkin [47. Our goal here is to give a more transparent proof, which is less obscured by peripheral matters, and which can be more easily adapted to the treatment of other problems as well.

Before proceeding it is appropriate to remind readers that Brown [7] was able to establish the existence of invariant subspaces for subnormal operators without actually addressing the BPE issue (cf. also [40]). 


\section{$\S 2$. The STRATEGY}

Our proof of Thomson's theorem will proceed generally along lines introduced in $[5]$ in order to obtain the same result for a large class of absolutely continuous measures. Due to its importance for our overall point of view we first recall here the main points of the earlier approach. For the remainder of this discussion $\mu$ will be a fixed positive measure of compact support in the complex plane $\mathbb{C}$, and $d A$ will denote two-dimensional Lebesgue measure. The letters $C$ and $K$, unless otherwise indicated, will be used to denote various absolute constants which may differ from one another, even within a single string of estimates.

As in the introduction suppose that $g \in L^{2}(d \mu)$, that $\int P g d \mu=0$ for every polynomial $P$, and form the Cauchy transform

$$
\widehat{g \mu}(\xi)=\int \frac{g(z)}{z-\xi} d \mu(z) .
$$

Fix a point $\xi_{0} \in \mathbb{C}$, and for each $\lambda>0$ consider the set $E_{\lambda}=\{z:|\widehat{g \mu}(z)|<\lambda\}$, which is precisely defined up to a set of $d A$-measure zero. There are two mutually exclusive possibilities: either

(3) $H^{2}(d \mu)$ has a BPE at $\xi_{0}$, or

(4) almost every circle $\left|z-\xi_{0}\right|=r$ meets each $E_{\lambda}$ in a set of positive linear measure.

To see that at least one of these must occur assume contrary to (4) that there exists a set $X$ of nonnegative real numbers having positive linear measure, such that $|\widehat{g \mu}| \geq \lambda$ almost everywhere on $\left|z-\xi_{0}\right|=r$ for every $r \in X$. If we let $X^{*}$ denote the union of all circles $\left|z-\xi_{0}\right|=r$ corresponding to these values of $r$, then by the mean value theorem

$$
P\left(\xi_{0}\right)=C \int_{X^{*}} P(\xi) d A_{\xi}
$$

for a suitable constant $C$ and all polynomials $P$. Expressing the integrand in accordance with the formula in (1.2) yields the representation

$$
\begin{aligned}
P\left(\xi_{0}\right) & =C \int_{X^{*}} \frac{1}{\widehat{g \mu}(\xi)}\left(\int \frac{P(z) g(z)}{(z-\xi)} d \mu_{z}\right) d A_{\xi} \\
& =C \int P(z) g(z)\left(\int_{X^{*}} \frac{1}{\widehat{g \mu}(\xi)} \frac{1}{(z-\xi)} d A_{\xi}\right) d \mu_{z} .
\end{aligned}
$$

Because $|\widehat{g \mu}| \geq \lambda$ almost everywhere on $X^{*}$ and $(z-\xi)^{-1}$ is locally integrable,

$$
\left|P\left(\xi_{0}\right)\right| \leq C \int|P||g| d \mu
$$

and hence $\left|P\left(\xi_{0}\right)\right| \leq C\|P\|_{L^{2}(d \mu)}$. Thus, if $H^{2}(d \mu)$ has no BPEs, each of the sets $E_{\lambda}$ is relatively massive near every point of $\mathbb{C}$. Our problem is to use this fact to conclude that $\widehat{g \mu}=0$ a.e.- $d A$.

In case $d \mu=w d A$ with $w \in L^{1+\epsilon}(d A)$ this can be done by appealing to essentially real variable methods. Since, in addition to the restriction on $w$, the function $g$ belongs to $L^{2}(w d A)$ it follows that the Cauchy transform $\widehat{g w}(=\widehat{g w d A})$ lies in a Sobolev space $W_{1}^{q}$ for some $q>1$ (cf. [5, p. 408]). As such, $\widehat{g w}$ enjoys a certain degree of continuity which is best described in terms of capacity, and, for the purpose of this study it can be assumed that $q \leq 2$, since otherwise every $W_{1}^{q}$ function is actually Hölder continuous or, more precisely, has a Hölder continuous representative. Nothing in the way of generality is lost if we further assume that $q<2$. 
If $1 \leq q<2$ the $q$-capacity of a compact planar set $E$ is by definition

$$
\Gamma_{q}(E)=\inf \int|\nabla u|^{q} d A
$$

the infimum being taken over all infinitely differentiable functions $u$ of compact support with $u \equiv 1$ on $E$. The $q$-capacity of an arbitrary set $X$ is defined to be

$$
\Gamma_{q}(X)=\sup \Gamma_{q}(E)
$$

where the supremum is taken over all compact sets $E \subseteq X$. If $X$ is a Borel set, it can be shown that

$$
\Gamma_{q}(X)=\inf \Gamma_{q}(G),
$$

where now the infimum is taken over all open sets $G \supseteq X$. A property is said to hold $q$-quasieverywhere if the set where it fails has $q$-capacity zero.

It is often convenient to have a different (but equivalent) notion of capacity. If $\nu$ is a positive Borel measure in $\mathbb{C}=\mathbb{R}^{2}$, let

$$
U^{\nu}(x)=\int|x-y|^{-1} d \nu(y)
$$

be the corresponding Newtonian potential; if $d \nu=f d A$, write $U^{f}(x)$ instead. For an arbitrary set $E$ define

$$
C_{q}(E)=\inf \int f^{q} d A
$$

the infimum being extended over all nonnegative functions $f \in L^{q}\left(\mathbb{R}^{2}, d A\right)$ such that $U^{f}(x) \geq 1$ on $E$. If $E$ is a Borel set and if $1<q<2$, it can be shown that

$$
C_{q}(E)^{1 / q}=\sup _{\nu} \nu(E)
$$

with the supremum being over all positive measures $\nu$ concentrated on $E$ for which $\left\|U^{\nu}\right\|_{L^{p}(d A)} \leq 1$. It is also known that the two capacities $\Gamma_{q}$ and $C_{q}$ are equivalent in the sense that there exists a constant $K>0$ such that

$$
K^{-1} C_{q}(E) \leq \Gamma_{q}(E) \leq K C_{q}(E)
$$

for every $E$. We will denote this (and similar equivalences) by writing $C_{q} \approx \Gamma_{q}$. For additional information and background material on capacity the reader is referred to the two books [1] and [24] where, in particular, proofs of the following can be found (cf. also [37] and [5, p. 411]):

(i) if $\Phi$ is a contraction, $C_{q}(\Phi E) \leq C_{q}(E)$;

(ii) $C_{q}\left(B_{r}\right) \approx C_{q}\left(\operatorname{diam} B_{r}\right) \approx r^{2-q}$ for any disk $B_{r}$ of radius $r$.

If $k \in L^{q}(d A)$ and $\int P k d A=0$ for every polynomial $P$, then $\hat{k}$ has compact support and, by the Calderón-Zygmund theorem on the continuity of singular integral operators (cf. [39, p. 35]),

$$
\|\nabla \hat{k}\|_{q} \leq C\left\|\frac{\partial \hat{k}}{\partial \bar{z}}\right\|_{q}=C \pi\|k\|_{q}
$$

provided that $q>1$, and so in this case $\hat{k} \in W_{1}^{q}$. Here the norms are taken with respect to area, that is, in $L^{q}(d A)$. For any $\lambda>0$ we also have a weak-type inequality

$$
\Gamma_{q}\{z \in \mathbb{C}:|\hat{k}(z)|>\lambda\} \leq \frac{C}{\lambda^{q}} \int|\nabla \hat{k}|^{q} d A,
$$


and this is the key to ascertaining the degree of continuity enjoyed by $\hat{k}$. If $\hat{k}_{j}=\hat{k} * \chi_{j}$ is a sequence of mollifiers obtained by convolving $\hat{k}$ with a $C^{\infty}$ approximate identity $\chi_{j}$, $j=1,2,3, \ldots$, it is well known that

$$
\left\|\hat{k}_{j}-\hat{k}\right\|_{q} \rightarrow 0 \quad \text { and } \quad\left\|\nabla \hat{k}_{j}-\nabla \hat{k}\right\|_{q} \rightarrow 0 .
$$

A careful analysis of the situation now reveals that, by passing to a subsequence if necessary, we can arrange for $\hat{k}_{j} \rightarrow \hat{k}$ uniformly off open sets of arbitrary small $q$-capacity (cf. [12, p. 354] and [50, p. 124]). The unavoidable conclusion is this: Given any $\epsilon>0$ there exists an open set $U$ such that $\Gamma_{q}(U)<\epsilon$ and $\hat{k}$ is continuous in the complement of $U$. Functions which have this property are said to be q-quasicontinuous. It is a fact that every $W_{1}^{q}$ function agrees almost everywhere $d A$ with one that is quasicontinuous. If $q>2$, then $\hat{k}$ is actually continuous and we have a corresponding assertion about $W_{1}^{q}$.

In addition to quasicontinuity there is a much subtler pointwise notion of continuity associated with functions in $W_{1}^{q}$, called fine continuity. A function $h$ that is defined $q$-quasieverywhere is said to be $q$-finely continuous at a point $x_{0}$ if there exists a set $E$ that is thin, or sparse, in a potential-theoretic sense at $x_{0}$ and

$$
\lim _{\substack{x \rightarrow x_{0} \\ x \in \mathbb{C} \backslash E}} h(x)=h\left(x_{0}\right) .
$$

The precise sense in which $E$ is understood to be thin is this: for $1<q \leq 2$, a set $E$ is $q$-thin at $x_{0}$ if and only if

$$
\int_{0}\left(\frac{\Gamma_{q}\left(E \cap B_{r}\right)}{r^{2-q}}\right)^{p-1} \frac{d r}{r}<\infty .
$$

Here $B_{r}=B\left(x_{0}, r\right)$ is the disk of radius $r$ with center at $x_{0}$ and $p=q /(q-1)$ is the index conjugate to $q$. If $E$ is not thin at $x_{0}$ it is said to be thick at that point. It is a fact that every $q$-quasicontinuous function is $q$-finely continuous $q$-q.e. (11, p. 177]). Because $C_{q}$ is countably subadditive ([1, p. 26]) and $\Gamma_{q} \approx C_{q}$ it follows as a corollary to (2.1) that if

$$
\limsup _{r \rightarrow 0} \frac{\Gamma_{q}\left(E \cap B_{r}\right)}{r^{2-q}}>0 \text {, }
$$

then $E$ is thick at $x_{0}$.

Let us now return to the situation described earlier where $g w \in L^{q}(d A), q>1$, and for every $\lambda>0$ and $\xi_{0} \in \mathbb{C}$ almost every circle $\left|z-\xi_{0}\right|=r$ meets $E_{\lambda}=\{z:|\widehat{g w}(z)|<\lambda\}$ in a set of positive linear measure. Since circular projection about any point $\xi_{0}$ onto a radial segment is a contraction, it can be inferred from (i) and (ii) that $\Gamma_{q}\left(E_{\lambda} \cap B_{r}\right) \approx r^{2-q}$ in all cases, and from (2.2), $E_{\lambda}$ is everywhere thick. Hence, by fine continuity, $|\widehat{g w}| \leq \lambda q$-q.e., and therefore a.e.- $d A$. Since this holds for every $\lambda>0$ we have shown that $\widehat{g w}=0$ a.e.$d A$, from which the bounded point evaluation assertion for $H^{2}(w d A)$ follows whenever $w \in L^{1+\epsilon}(d A)$.

A similar argument (cf. [5, 23]) yields the same conclusion under the weaker assumption that

$$
\int w\left(\log ^{+} w\right)^{2} d A<\infty .
$$

Under that restriction on $w$, if $g \in L^{2}(w d A)$ and $k=g w$, then

$$
\int|k|\left(1+\log ^{+}|k|\right) d A<\infty
$$

and so, by a theorem of Calderón and Zygmund, $\nabla \hat{k} \in L^{1}(d A)$ (cf. [39, p. 48]). That is, $\hat{k} \in B V$, the space consisting of all functions having bounded variation in the sense of DeGiorgi. Should $g$ happen to be orthogonal to the polynomials in $L^{2}(w d A)$, then, 
in the absence of BPEs, $\hat{k}=\widehat{g w}$ necessarily enjoys property (4) above. By once again considering projections of the corresponding sets $E_{\lambda}$ onto linear segments, this is easily seen to be incompatible with $\hat{k} \in B V$, unless $\hat{k}=0$ a.e.- $d A$. The argument here can also be recast in terms of a capacity introduced by Fleming [13, and designed to more accurately describe the exceptional sets associated with $B V$ functions.

If we assume only that $w \in L^{1}(d A)$ the line of reasoning pursued above fails in at least one important respect. In this case no useful estimate for thickness can be obtained by projecting $E_{\lambda}$ onto a line segment, since the 1-capacity of any such segment is zero. On the other hand, nowhere have we made use heretofore of the fact that almost every circle $\left|z-\xi_{0}\right|=r$ meets $E_{\lambda}$ in a set of positive linear measure. In order to establish the general theorem it will be essential to bring this phenomenon to bear on the problem.

\section{§3. Analytic CAPACity}

The concept of analytic capacity was introduced by Ahlfors 2] in 1947 in connection with the problem of characterizing sets of removable singularities for bounded analytic functions, otherwise known as the Painlevé problem. In the ensuing years others, and Vitushkin in particular, further developed the concept and used it to settle a number of questions concerning uniform approximation by rational functions on compact subsets of the plane. An extensive and in-depth discussion of analytic capacity and its many applications covering the period from 1950 to the turn of the century can be found collectively in the expository articles of Vitushkin [47, Mel'nikov and Sinanyan [33, and Khavinson [20] and in the monographs of Gamelin [14, Garnett [16], and Zalcman [49] (cf. also Conway [10] and Davie [11]).

In keeping with established custom the letters $X$ and $K$ will be used throughout this section to represent compact subsets of the complex plane $\mathbb{C}$; and $\hat{\mathbb{C}}$ will stand for the extended plane or Riemann sphere. The analytic capacity of a compact set $X$, denoted $\gamma(X)$, is defined as follows:

$$
\gamma(X)=\sup \left|f^{\prime}(\infty)\right|
$$

where the supremum is extended over all functions $f$ analytic in $\hat{\mathbb{C}} \backslash X$ and normalized so that

(a) $\|f\|_{\infty}=\sup _{\hat{\mathbb{C}} \backslash X}|f| \leq 1$,

(b) $f(\infty)=0$.

In this case there exists a unique admissible $f$ with $f^{\prime}(\infty)=\gamma(X)$. For an arbitrary planar set $E$ we let $\gamma(E)=\sup \gamma(X)$, the supremum now being taken over all compact sets $X \subseteq E$. For a more thorough discussion of analytic capacity and its properties see [14] where, for instance, it is shown that

(i) $\gamma\left(B_{r}\right)=r$ for every disk $B_{r}$ of radius $r$;

(ii) $\gamma(X) \approx \operatorname{diam}(X)$ whenever $X$ is compact and connected; in particular, $\gamma(X) \leq$ $\operatorname{diam}(X) \leq 4 \gamma(X)$.

Although there are similarities between analytic capacity and the potential-theoretic capacities of Section 2, there are also some significant differences. Two differences that make it impossible to directly extend our earlier reasoning on the bounded point evaluation problem to the general case are these: unlike $q$-capacity $C_{q}$,

(iii) $\gamma$ is not known to be subadditive;

(iv) if $\Phi$ is a contraction, it may not happen that $\gamma(\Phi E) \leq \gamma(E)$.

The phenomenon referred to in the second assertion was first observed by Vitushkin [46] in 1959. In that paper he constructed a Cantor set $E$ with the property that $\gamma(E)=0$, and so that its orthogonal projection $\Phi E$ onto some line is a nondegenerate interval; that is, $\gamma(\Phi E)>0$. Hence, there is in general no constant $C>0$ such that 
$\gamma(\Phi E) \leq C \gamma(E)$. Vitushkin's example was eventually simplified by Garnett [15] and Ivanov [22, pp. 346-348].

The situation with regard to subadditivity is not quite as serious, however. Tolsa 44] has recently shown that analytic capacity $\gamma$ is semiadditive in the sense that

$$
\gamma(E \cup F) \leq C(\gamma(E)+\gamma(F))
$$

for all compact sets $E, F \subseteq \mathbb{C}$ and some absolute constant $C$. The key consists in showing that $\gamma$ is equivalent to another capacity $\gamma^{+}$, which is known to be semiadditive, and which for our purpose is more directly linked to the Cauchy integral. For a compact set $X$ we define

$$
\gamma^{+}(X)=\sup _{\nu} \nu(X)
$$

where the supremum is taken over all positive measures $\nu$ supported on $X$ such that $\hat{\nu} \in L^{\infty}(\mathbb{C})$ and $\|\hat{\nu}\|_{\infty} \leq 1$. Since $\hat{\nu}$ is analytic in $\mathbb{C} \backslash X$ and $\hat{\nu}^{\prime}(\infty)=\nu(X)$, the function $\hat{\nu}$ is admissible in the definition of $\gamma$ and thus

$$
\gamma^{+}(X) \leq \gamma(X) .
$$

As before, if $E$ is an arbitrary planar set we let

$$
\gamma^{+}(E)=\sup _{X} \gamma^{+}(X)
$$

where $X$ is compact and $X \subseteq E$.

Tolsa's theorem (cf. 44), which provides a solution to the Painlevé problem and affirms an old conjecture of Vitushkin, has its roots in the work of Mattila, Mel'nikov and Verdera [27, 31, 34] and is as follows.

Theorem 2. There exists an absolute constant $C>0$ so that

$$
\gamma^{+}(E) \leq \gamma(E) \leq C \gamma^{+}(E)
$$

for all sets $E \subseteq \mathbb{C}$; that is, $\gamma \approx \gamma^{+}$.

Since Tolsa [42] had previously shown that $\gamma^{+}$is in fact countably semiadditive, the same is true for $\gamma$ :

(1) If $E_{n}, n=1,2,3, \ldots$ are Borel sets, then

$$
\gamma\left(\bigcup_{n} E_{n}\right) \leq C \sum_{n} \gamma\left(E_{n}\right),
$$

where $C$ is again an absolute constant.

There is also a weak-type estimate for the Cauchy integral which is suggestive of a kind of pointwise continuity similar to that enjoyed by ordinary potentials, but a corresponding phenomenon has yet to be established in this context (cf. [43]):

(2) If $\mu$ is a complex measure and $\hat{\mu}(x)$ is taken in the principal value sense, then for any $\lambda>0$,

$$
\gamma\{x \in \mathbb{C}:|\hat{\mu}(x)|>\lambda\}<\frac{C}{\lambda}\|\mu\|,
$$

where $\|\mu\|$ is the total variation of the measure $\mu$.

An exposition of many of the details associated with these results and a discussion of the Painlevé problem in general can be found in the Lecture Notes of Pajot [38. See also the expository articles [9, 26, 32, 45].

In order to settle the bounded point evaluation question along the lines indicated earlier in Section 2 we need a substitute for the notion of fine continuity, a concept which is applicable to potentials but is, so far, lacking in the case of the Cauchy integral. A substitute is provided by the following two lemmas. Since they are similar in nature we 
shall present a detailed proof of the first and simply indicate the necessary modifications in the proof of the second. Each can be viewed as a variation of a prior result of the author (cf. [4, Lemma 2]), and each was suggested by an idea of Carleson (cf. 8, Lemma 1]) used to give a short proof of Mergelyan's theorem on uniform polynomial approximation.

Lemma 1. Let $\nu$ be a finite positive Borel measure of compact support in the complex plane $\mathbb{C}$ with the property that $|\hat{\nu}(z)| \leq C$ a.e.-dA for some constant $C$. Then $\left|\hat{\nu}\left(x_{0}\right)\right| \leq C$ at every point $x_{0}$ where $U^{\nu}\left(x_{0}\right)=\int \frac{d \nu(\zeta)}{\left|\zeta-x_{0}\right|}<\infty$.

Lemma 2. Let $\mu$ be a finite complex, compactly supported, Borel measure in $\mathbb{C}$, and let $x_{0}$ be any point where $U^{|\mu|}\left(x_{0}\right)<\infty$. For each $r>0$ let $B_{r}=B\left(x_{0}, r\right)$ be the disk with center at $x_{0}$ and radius $r$, and let $E$ be a set with the property that for every $r>0$ there is a relatively large subset $E_{r} \subseteq\left(E \cap B_{r}\right)$ on which $U^{|\mu|}$ is bounded; that is,

(1) $U^{|\mu|} \leq M_{r}<\infty$ on $E_{r}$,

(2) $\gamma\left(E_{r}\right) \geq \epsilon \gamma\left(E \cap B_{r}\right)$ for some absolute constant $\epsilon$.

If, moreover, $E$ is thick at $x_{0}$ in the sense that

$$
\limsup _{r \rightarrow 0} \frac{\gamma\left(E \cap B_{r}\right)}{r}>0
$$

then $\left|\hat{\mu}\left(x_{0}\right)\right| \leq \lim \sup _{z \rightarrow x_{0}, z \in E}|\hat{\mu}(z)|$.

Proof of Lemma 1. We may assume that $x_{0}=0$ so that $U^{\nu}(0)=\int \frac{d \nu(z)}{|z|}<\infty$. For each $r>0$ let $B_{r}=B(0, r)$ be the disk of radius $r$ with center at the origin, let $\chi_{r}$ be the characteristic function of $B_{r}$, and define a probability measure $\sigma_{r}$ on $B_{r}$ by setting $d \sigma_{r}=\frac{1}{\pi r^{2}} \chi_{r} d A$. We shall verify that

$$
\lim _{r \rightarrow 0} \int \hat{\nu}(\zeta) d \sigma_{r}(\zeta)=\hat{\nu}(0)
$$

from which it follows that

$$
|\hat{\nu}(0)| \leq \limsup _{r \rightarrow 0} \int|\hat{\nu}| d \sigma_{r} \leq C,
$$

since $|\hat{\nu}| \leq C$ a.e.- $d \sigma_{r}$ for each $r$.

In order to establish (3.3) we begin with an interchange in the order of integration which gives

$$
\int \hat{\nu}(\zeta) d \sigma_{r}(\zeta)=\int_{|z|<2 r}+\int_{|z| \geq 2 r}\left\{\int \frac{d \sigma_{r}(\zeta)}{z-\zeta}\right\} d \nu(z)
$$

The interchange is justified by virtue of the fact that $\int \frac{d \sigma_{r}(\zeta)}{|z-\zeta|} \leq \frac{2}{r}$ for all $z \in \mathbb{C}$, and so the iterated integral on the right converges absolutely. It is then an easy matter to check that

(i) $\int \frac{d \sigma_{r}(\zeta)}{z-\zeta} \rightarrow \frac{1}{z}$ for every $z \neq 0$ as $r \rightarrow 0$,

(ii) $\int \frac{d \sigma_{r}(\zeta)}{|z-\zeta|} \leq \frac{2}{|z|}$ for $|z| \geq 2 r$.

By assumption $\int \frac{d \nu(z)}{|z|}<\infty$, and so the dominated convergence theorem implies that

$$
\lim _{r \rightarrow 0} \int_{|z| \geq 2 r}\left\{\int \frac{d \sigma_{r}(\zeta)}{z-\zeta}\right\} d \nu(z)=\int \frac{d \nu(z)}{z}=\hat{\nu}(0) .
$$

On the other hand,

$$
\left|\int_{|z|<2 r}\left\{\int \frac{d \sigma_{r}(\zeta)}{z-\zeta}\right\} d \nu(z)\right| \leq \int_{|z|<2 r} \frac{2}{r} d \nu(z) \leq \int_{|z|<2 r} \frac{4}{|z|} d \nu(z),
$$


and the last integral tends to zero as $r \rightarrow 0$ by our initial assumption. This establishes (3.3) and the lemma as well.

Proof of Lemma 2. Assume as above that $x_{0}=0$. For some sequence of $r \rightarrow 0$ we shall construct a corresponding sequence of probability measures $\nu_{r}$ such that

(a) $\nu_{r}$ is carried by $E \cap B_{r}$,

(b) $\lim _{r \rightarrow 0} \int \hat{\mu} d \nu_{r}=\hat{\mu}(0)$.

Once this has been accomplished the desired conclusion is immediate:

$$
|\hat{\mu}(0)| \leq \limsup _{z \rightarrow 0, z \in E}|\hat{\mu}(z)| .
$$

In order to obtain the measures $\nu_{r}$ we make use of the fact that $\gamma \approx \gamma^{+}$, and so each of the hypotheses in the lemma remains valid for $\gamma^{+}$with possibly different constants. In particular, according to (3.2),

$$
\limsup _{r \rightarrow 0} \frac{\gamma^{+}\left(E \cap B_{r}\right)}{r}>0
$$

Thus, there is a constant $C>0$ and a sequence of $r \rightarrow 0$ such that $\gamma^{+}\left(E_{r}\right)>C r$ for each corresponding $r$. Consistent with the definition of $\gamma^{+}$, we can then select a positive measure $\sigma_{r}$ on $E_{r}$ with

(c) $\left\|\sigma_{r}\right\|=\sigma_{r}\left(E_{r}\right) \geq C r$,

(d) $\left|\hat{\sigma}_{r}\right| \leq 1$ a.e.- $d A$.

Setting $\nu_{r}=\frac{\sigma_{r}}{\left\|\sigma_{r}\right\|}$ we obtain a probability measure on $E_{r} \subseteq\left(E \cap B_{r}\right)$, and $\left|\hat{\nu}_{r}\right| \leq \frac{C}{r}$ a.e.- $d A$ for some absolute constant $C$. As in the proof of the preceding lemma,

(i) $\int \frac{d \nu_{r}(\zeta)}{z-\zeta} \rightarrow \frac{1}{z}$ for every $z \neq 0$ as $r \rightarrow 0$,

(ii) $\int \frac{d \nu_{r}(\zeta)}{|z-\zeta|} \leq \frac{2}{|z|}$ for $|z| \geq 2 r$.

Because $U^{|\mu|} \leq M_{r}$ on $E_{r}$, it follows from Fubini's theorem that

$$
\int\left(\int \frac{d \nu_{r}(\zeta)}{|z-\zeta|}\right) d|\mu|(z)=\int\left(\int \frac{d|\mu|(z)}{|z-\zeta|}\right) d \nu_{r}(\zeta) \leq M_{r}
$$

and hence $U^{\nu_{r}}<\infty$ a.e.- $d|\mu|$. Therefore, by Lemma $1,\left|\hat{\nu}_{r}(z)\right| \leq \frac{C}{r}$ a.e.-d $d|\mu|$.

We can now proceed exactly as in the proof of Lemma 1, writing

$$
\int \hat{\mu}(\zeta) d \nu_{r}(\zeta)=\int_{|z|<2 r}+\int_{|z| \geq 2 r}\left\{\int \frac{d \nu_{r}(\zeta)}{z-\zeta}\right\} d \mu(z)
$$

As a consequence of (i) and (ii) we have

$$
\lim _{r \rightarrow 0} \int_{|z| \geq 2 r}\left\{\int \frac{d \nu_{r}(\zeta)}{z-\zeta}\right\} d \mu(z)=\int \frac{d \mu(z)}{z}=\hat{\mu}(0) .
$$

For the remaining integral over $|z|<2 r$ we have the estimate

$$
\left|\int_{|z|<2 r}\left\{\int \frac{d \nu_{r}(\zeta)}{z-\zeta}\right\} d \mu(z)\right| \leq \int_{|z|<2 r} \frac{C}{r} d|\mu|(z) \leq 2 C \int_{|z|<2 r} \frac{d|\mu|(z)}{|z|},
$$

and the last integral tends to zero as $r \rightarrow 0$ by our assumption that $U^{|\mu|}(0)<\infty$. This establishes property (b), and the lemma follows.

In some instances, especially in those of particular interest here, verification of the density criterion (3.2) requires the introduction of a third auxiliary capacity $\tilde{\gamma}$ defined as follows: if $X \subseteq \mathbb{C}$ is compact,

$$
\tilde{\gamma}=\sup |\mu(X)|=\sup \left|f^{\prime}(\infty)\right|,
$$


where the supremum is taken over all absolutely continuous measures $d \mu=g d A$ supported on $X$ with $f=\hat{g}$ and $\|f\|_{\infty} \leq 1$. For an arbitrary measurable set $E$ we define

$$
\tilde{\gamma}(E)=\sup _{X} \tilde{\gamma}(X)
$$

where $X$ is compact and $X \subseteq E$. It is an immediate consequence of the definition that

$$
\gamma(E) \geq \tilde{\gamma}(E)
$$

for all measurable sets $E$, and consideration of the function $f(z)=\int_{E} \frac{d A_{\zeta}}{\zeta-z}$ yields the estimate

$$
\tilde{\gamma}(E) \geq \sqrt{\frac{A(E)}{4 \pi}} .
$$

For a proof of the latter, see [14, p. 200]. Capacities similar to $\tilde{\gamma}$ have been studied for many years, beginning with Khavinson [19] (cf. 20] for a brief survey of results prior to 1999).

Consider now an application of the Vitushkin approximation scheme due to Mel'nikov 30. For each positive integer $n$ form a grid in the plane consisting of lines parallel to the coordinate axes, intersecting at those points whose coordinates are both integral multiples of $2^{-n}$. The resulting collection of squares $\mathcal{G}_{n}=\left\{S_{n j}\right\}_{j=1}^{\infty}$ of side lengths $2^{-n}$ is an edge-to-edge tiling of the entire plane; its members will be referred to as squares of the $n$-th generation. Of chief concern here are tilings made up of squares from several generations. Beginning with a fixed positive integer $k$, let $\Pi_{k}$ be a polygon formed by taking the union of finitely many squares from $\mathcal{G}_{k}$, and denote its boundary by $\Gamma_{k}$. Next, adjoin to $\prod_{k}$ finitely many additional squares from the next generation $\mathcal{G}_{k+1}$ extending outward from $\Pi_{k}$ and separating $\Gamma_{k}$ from $\infty$ to obtain a second polygon $\Pi_{k+1}$ bounded by $\Gamma_{k+1}$. Continuing in this way we construct a finite sequence of polygons

$$
\Pi_{k} \subseteq \Pi_{k+1} \subseteq \cdots \subseteq \Pi_{k+l},
$$

and we shall assume at the final stage that $\prod_{k+l}$ extends to $\infty$ in all directions. Collectively, the squares in any such sequence, and hence those at the top of the chain, can be viewed as a kind of tiling of the plane. Enlarging each square $S_{r}$ occurring in (3.5) by a factor of $5 / 4$ we obtain an open covering of the plane such that no point $z \in \mathbb{C}$ lies in more than 4 of the corresponding enlarged squares $Q_{r}$. Except for minor modifications, the next lemma is due to Mel'nikov (cf. [30, Assertion 1]). For ease of notation we shall henceforth denote the area of a set $E$ by $|E|$ in place of the earlier designation $A(E)$.

Lemma 3. Suppose that a nested sequence of polygons

$$
\Pi_{k} \subseteq \Pi_{k+1} \subseteq \cdots \subseteq \Pi_{k+l}
$$

has been constructed in the manner described above. Let $K=\bigcup K_{n}$ be a compact set where for each $n$,

(1) $K_{n}=\bigcup S_{n j}$ is a finite union of closed squares $S_{n j}$ in $\Pi_{n} \backslash \Pi_{n-1}$, and

(2) $n^{2} 2^{-n} \leq \operatorname{dist}\left(K_{n+1}, \Pi_{n}\right) \leq 3 n^{2} 2^{-n}$.

If $E \subseteq K$ is a measurable set with the property that $\left|E \cap S_{n j}\right|>\epsilon\left|S_{n j}\right|$ for some fixed constant $\epsilon>0$ and all $S_{n j} \subseteq K$, then

$$
\tilde{\gamma}(E) \geq C \epsilon \gamma(K),
$$

where $C$ is an absolute constant.

Proof. Let $f$ be the Ahlfors function for $K$; that is, $f$ is analytic outside of $K,\|f\|_{\infty} \leq 1$, $f(\infty)=0$, and $f^{\prime}(\infty)=\gamma(K)$. By modifying $f$ in a small neighborhood of $K$ we can assume that $f$ is continuous on $\hat{\mathbb{C}}$. 
The collection of all open squares $Q_{n j}$ obtained by enlarging those in the tiling $\Pi_{k+l}$ is an open cover of the plane with no point $z \in \mathbb{C}$ lying in more than 4 squares. Here, each $Q_{n j}$ has side length $\frac{5}{4} \delta_{n}$ with $\delta_{n}=2^{-n}$ being the side length of $S_{n j}$. We can therefore choose continuously differentiable functions $g_{n j}$ such that

(i) $g_{n j}$ is supported in $Q_{n j}$,

(ii) $\sum g_{n j}(z)=1$ for all $z \in \mathbb{C}$,

(iii) $\left\|\operatorname{grad} g_{n j}\right\|_{\infty} \leq \frac{100}{\delta_{n}}$

(cf., for example, [11, p. 417]). Setting

$$
\begin{aligned}
G_{n j}(z) & =\frac{1}{\pi} \int \frac{f(\zeta)-f(z)}{\zeta-z} \frac{\partial g_{n j}}{\partial \bar{\zeta}} d A_{\zeta} \\
& =f(z) g_{n j}(z)+\frac{1}{\pi} \int \frac{f(\zeta)}{\zeta-z} \frac{\partial g_{n j}}{\partial \bar{\zeta}} d A_{\zeta}
\end{aligned}
$$

we obtain functions $G_{n j}$ which are analytic off $Q_{n j}$ and analytic everywhere that $f$ is analytic. It follows from (ii) that $f=\sum G_{n j}$, and the sum is finite since $G_{n j}=0$ except for those indices for which $Q_{n j}$ meets $K$.

By assumption, and in view of (3.4), for these indices we have the lower estimate

$$
\tilde{\gamma}\left(E \cap S_{n j}\right)>\sqrt{\frac{\epsilon}{4 \pi}} \delta_{n} .
$$

Indeed, for each square $S_{n j}$ choose a compact set $E_{n j} \subseteq\left(E \cap S_{n j}\right)$ with $\left|E_{n j}\right|>\epsilon \delta_{n}^{2}$, and let

$$
\psi_{n j}(z)=\sqrt{\frac{\epsilon}{4 \pi}} \delta_{n} \frac{1}{\left|E_{n j}\right|} \int_{E_{n j}} \frac{d A_{\zeta}}{\zeta-z} .
$$

Thus, $\psi_{n j}$ is analytic off $E_{n j}, \psi_{n j}(\infty)=0,\left\|\psi_{n j}\right\|_{\infty} \leq 1$, and $\left|\psi_{n j}^{\prime}(\infty)\right|=\sqrt{\frac{\epsilon}{4 \pi}} \delta_{n}$. Expanding $\psi_{n j}$ in a Laurent series about $z_{n j}$, the center of $S_{n j}$, we have

$$
\psi_{n j}(z)=\frac{a_{1}}{\left(z-z_{n j}\right)}+\frac{a_{2}}{\left(z-z_{n j}\right)^{2}}+\cdots
$$

valid everywhere outside the enlarged square $Q_{n j}$. Here, $\left|a_{1}\right|=\sqrt{\frac{\epsilon}{4 \pi}} \delta_{n}$, and it is easily checked that $\left|a_{2}\right| \leq \sqrt{\frac{\epsilon}{8 \pi}} \delta_{n}^{2}$. The bounds on $\left|a_{1}\right|$ and $\left|a_{2}\right|$ allow us to construct functions $H_{n j}$ in such a way that

(iv) $H_{n j}(z)=\int_{E_{n j}} \frac{h_{n j}(\zeta)}{\zeta-z} d A_{\zeta}, h_{n j} \in L^{\infty}$

(v) $\left\|H_{n j}\right\|_{\infty} \leq \frac{C}{\epsilon}, C$ an absolute constant;

(vi) $\lim _{z \rightarrow \infty} z^{2}\left[G_{n j}(z)-H_{n j}(z)\right]=0$.

We have only to express $G_{n j}$ in a Laurent series

$$
G_{n j}(z)=\frac{b_{1}}{\left(z-z_{n j}\right)}+\frac{b_{2}}{\left(z-z_{n j}\right)^{2}}+\cdots
$$

and proceed as in Vitushkin [47, p. 153], setting

$$
H_{n j}=\frac{b_{1}}{a_{1}} \psi_{n j}+\frac{b_{2}}{a_{1}^{2}} \psi_{n j}^{2}-\frac{b_{1} a_{2}}{a_{1}^{3}} \psi_{n j}^{2} .
$$

Since $H_{n j}(\infty)=0$, property (iv) is satisfied with $h_{n j}=\bar{\partial} H_{n j}$, and (vi) is guaranteed by construction. Since $\left|b_{1}\right| \leq C_{1} \delta_{n}$ and $\left|b_{2}\right| \leq C_{2} \delta_{n}^{2}$ with $C_{1}$ and $C_{2}$ independent of $n$, condition (v) is also fulfilled. Hence,

$$
\left\|G_{n j}-H_{n j}\right\|_{\infty} \leq \frac{C}{\epsilon}
$$

by virtue of the fact that the $G_{n j}$ are uniformly bounded by a constant depending only on $f$. 
Setting $\phi=\sum H_{n j}$ and appealing to the fact that $H_{n j}^{\prime}(\infty)=G_{n j}^{\prime}(\infty)$ we conclude that $\phi^{\prime}(\infty)=f^{\prime}(\infty)=\gamma(K)$. If it can be shown that $\|\phi\|_{\infty} \leq \frac{C_{3}}{\epsilon}$ for some absolute constant $C_{3}$, the desired inequality

$$
\tilde{\gamma}(E) \geq C \epsilon \gamma(K)
$$

follows, since $\phi=\hat{h}$ where $h=\sum h_{n j}$ is in $L^{\infty}$ with support in $E$.

Toward this end note that according to (vi) the function $\left(z-z_{n j}\right)^{3}\left(G_{n j}-H_{n j}\right)$ is analytic off $Q_{n j}$ and so by the maximum principle

$$
\left|z-z_{n j}\right|^{3}\left|G_{n j}-H_{n j}\right| \leq \frac{C}{\epsilon}\left(\frac{5 \sqrt{2}}{8}\right)^{3} \delta_{n}^{3}
$$

everywhere outside and on the boundary of $Q_{n j}$. It follows that

$$
\left|G_{n j}(z)-H_{n j}(z)\right| \leq \frac{C_{4}}{\epsilon} \min \left\{1, \frac{\delta_{n}^{3}}{\left|z-z_{n j}\right|^{3}}\right\}
$$

for all $z \in \mathbb{C}$. In order to verify that $\|\phi\|_{\infty} \leq \frac{C_{3}}{\epsilon}$ it is enough, therefore, to show that

$$
\sum_{n, j} \min \left\{1, \frac{\delta_{n}^{3}}{\left|z-z_{n j}\right|^{3}}\right\} \leq C_{5}
$$

for some constant $C_{5}$ independent of $z$. We shall argue as in Davie [11, p. 417].

Fix a point $z \in \mathbb{C}$, and consider initially only those terms in the sum (3.6) corresponding to the centers $z_{n j}$ of squares from the $n$-th generation; that is, to the centers of the squares comprising $K_{n}$. Summing over the associated indices it can be shown that

$$
\sum_{j} \min \left\{1, \frac{\delta_{n}^{3}}{\left|z-z_{n j}\right|^{3}}\right\} \leq C_{6} \min \left\{1, \frac{2^{-n}}{\operatorname{dist}\left(z, \bigcup_{j} Q_{n j}\right)}\right\},
$$

where $C_{6}$ is a constant independent of $n$. To see this let $d=\operatorname{dist}\left(z, \bigcup_{j} Q_{n j}\right)$, and form the sequence of concentric annuli $A_{s}=\{w: s d<|w-z|<(s+1) d\}, s=0,1,2, \ldots$. The proof of (3.7) is as follows.

By a generous estimate there are no more than 25 squares $Q_{n j}$ for which $\operatorname{dist}\left(z, Q_{n j}\right) \leq$ $2^{-n}$; for all others $\left|z-z_{n j}\right|>d>2^{-n}$. Since each point is contained in at most 4 of the $Q_{n j}$

$$
\sum_{j} \min \left\{1, \frac{\delta_{n}^{3}}{\left|z-z_{n j}\right|^{3}}\right\} \leq 100+\sum_{\left|z-z_{n j}\right|>2^{-n}} \frac{2^{-3 n}}{\left|z-z_{n j}\right|^{3}} .
$$

Moreover, each square $Q_{n j}$ corresponding to a term in the sum on the right meets at least two and at most three of the annuli under consideration. Thus, if $N(s)$ denotes the number of squares $Q_{n j}$ meeting $A_{s}$, then

$$
N(s)\left(\frac{5}{4}\right)^{2} 2^{-2 n} \leq 4\left|A_{s} \cup A_{s+1} \cup A_{s+2}\right|=4 \pi d^{2}(6 s+9) .
$$

Hence, $N(s) \leq 200 \frac{d^{2}}{2^{-2 n}} s$ for all $s=1,2,3$, and it follows that

$$
\sum_{\left|z-z_{n j}\right|>2^{-n}} \frac{2^{-3 n}}{\left|z-z_{n j}\right|^{3}} \leq\left(200 \sum_{s=1}^{\infty} \frac{1}{s^{2}}\right) \frac{2^{-n}}{d} .
$$

Together (3.8) and (3.9) yield the desired estimate in (3.7). 
If $z \in \Pi_{q} \backslash \Pi_{q-1}$ for some $q$, then summing over all generations,

$$
\sum_{r} \min \left\{1, \frac{\delta_{r}^{3}}{\left|z-z_{r}\right|^{3}}\right\} \leq 3 C_{6}+C_{6} \sum_{|n-q| \geq 2} \frac{2^{-n}}{\operatorname{dist}\left(z, \bigcup_{j} Q_{n j}\right)} \leq 3 C_{6}+C_{7} \sum_{n=1}^{\infty} \frac{1}{n^{2}},
$$

since $\operatorname{dist}\left(z, \bigcup_{j} Q_{n j}\right) \approx \operatorname{dist}\left(z, K_{n}\right)$ and, by assumption, $\operatorname{dist}\left(z, K_{n}\right) \geq n^{2} 2^{-n}$ whenever $|n-q| \geq 2$. This establishes (3.6) and completes the proof of the lemma.

\section{$\S 4$. A SCHEME FOR $L^{2}$-APPROXimation}

We are now at a point where we can begin to address the bounded point evaluation problem in its most general context. Throughout the discussion $\mu$ will be a positive compactly supported measure in $\mathbb{C}$, and $g \in L^{2}(d \mu)$ will be an annihilator for $H^{2}(d \mu)$; that is,

$$
\int \operatorname{Pg} d \mu=0
$$

for all polynomials $P$. By convention, $\nu=g \mu$. The question of the existence of bounded point evaluations (BPEs) for $H^{2}(d \mu)$ is closely linked to an analogous question in the $L^{1}(|\hat{\nu}| d A)$ norm. This fact played a key role in our discussion of the BPE problem for absolutely continuous measures in Section 2. Our treatment of the general problem is based on the following elementary fact.

Lemma 4. If there is a BPE for the polynomials in the $L^{1}(|\hat{\nu}| d A)$ norm at a point $x_{0}$, then $H^{2}(d \mu)$ also has a BPE at $x_{0}$.

Proof. By assumption there exists a function $h \in L^{\infty}(d A)$ such that $P\left(x_{0}\right)=\int P h|\hat{\nu}| d A$ for every polynomial $P$. Letting $k=h \frac{|\hat{\nu}|}{\hat{\nu}}$ when $\hat{\nu} \neq 0$ we have $\|k\|_{\infty}=\|h\|_{\infty}$ and, by an interchange in the order of integration,

$$
P\left(x_{0}\right)=\int P k \hat{\nu} d A=-\int \widehat{P k} d \nu .
$$

On the other hand, $\widehat{P k}=P \hat{k}+F$, where $F$ is entire. Since $\nu$ annihilates the polynomials, and so also $F$ (that is, $\int F d \nu=0$ ), we can infer that

$$
P\left(x_{0}\right)=-\int P \hat{k} d \nu=-\int P \hat{k} g d \mu .
$$

By a straightforward application of Schwarz's inequality it follows that

$$
\left|P\left(x_{0}\right)\right| \leq K\|P\|_{L^{2}(d \mu)}
$$

for all polynomials $P$, and so $H^{2}(d \mu)$ has a BPE at $x_{0}$.

Our immediate task is to develop a procedure which, in the absence of BPEs for $H^{2}(d \mu)$, will allow us to conclude that $H^{2}(d \mu)=L^{2}(d \mu)$ along lines similar to those employed in Section 2. The scheme that we shall adopt is due, in broad outline, to Thomson [41], and has its roots in the work of Mel'nikov [30]. As in the special cases considered earlier, the sets $E_{\lambda}=\{z:|\hat{\nu}(z)|<\lambda\}$ will play a critical role.

Let $x_{0} \in \mathbb{C}$ be a point where $U^{|\nu|}\left(x_{0}\right)<\infty$, and fix $\lambda>0$. Beginning with a particular generation, the $n$-th say, choose a square $S^{*} \in \mathcal{G}_{n}$ with $x_{0} \in S^{*}$. Denote by $\mathcal{G}_{n}^{\lambda}$ the collection of all squares $S$ in $\mathcal{G}_{n}$ for which

$$
\left|E_{\lambda} \cap S\right|>\frac{1}{100}|S| .
$$

$K_{n}$ will stand for the union of those squares in $\mathcal{G}_{n}^{\lambda}$ that can be joined to $S^{*}$ by a finite chain of squares each lying in $\mathcal{G}_{n}^{\lambda}$. In the event that $K_{n}$ is bounded, or perhaps empty, there exists a closed corridor, or barrier, $Q_{n}=\bigcup_{j} S_{n j}$ composed of squares $S_{n j}$ from 
the $n$-th generation $\mathcal{G}_{n}$ abutting $S^{*} \cup K_{n}$, separating the latter from $\infty$, adjacent to one another along their sides, and such that

$$
\left|E_{\lambda} \cap S_{n j}\right| \leq \frac{1}{100}\left|S_{n j}\right|
$$

for each $j$. The polynomially convex hull of $Q_{n}$ is a polygon $\Pi_{n}$ with its boundary $\Gamma_{n}$ lying along the sides of squares for which (4.2) is satisfied. In other words, $|\hat{\nu}| \geq \lambda$ on a large percentage of every square $S_{n j}$ meeting $\Gamma_{n}$.

Next, construct a polygon $\Pi_{n}^{*}$ with boundary $\Gamma_{n}^{*}$ such that

(i) $\Pi_{n}^{*} \supseteq \Pi_{n}$,

(ii) $n^{2} 2^{-n} \leq \operatorname{dist}\left(\Gamma_{n}^{*}, \Gamma_{n}\right) \leq 3 n^{2} 2^{-n}$.

This can be done by simply adjoining to $\prod_{n}$ additional squares from $\mathcal{G}_{n}$. Let $K_{n+1}$ denote the union of all squares in $\mathcal{G}_{n+1}^{\lambda}$ that can be joined to $\Pi_{n}^{*}$ by a finite chain of squares in $\mathcal{G}_{n+1}^{\lambda}$. If $K_{n+1}$ is bounded, or empty, there is a second barrier $Q_{n+1}$ abutting $\Pi_{n}^{*} \cup K_{n+1}$ such that

$$
\left|E_{\lambda} \cap S\right| \leq \frac{1}{100}|S|
$$

for every square $S$ in $Q_{n+1}$. The polygon $\Pi_{n+1}$ is taken to be the polynomially convex hull of $Q_{n+1}$, its boundary is $\Gamma_{n+1}$, and the process continues.

The result of the construction is a nested sequence of polygons

$$
\Pi_{n} \subseteq \Pi_{n+1} \subseteq \cdots \subseteq \Pi_{n+l} \subseteq \cdots
$$

and compact sets $K_{j} \subseteq \Pi_{j}, j \geq n$, for which the hypotheses of Lemma 3 are satisfied; that is,

(i) $K_{j} \subseteq \Pi_{j} \backslash \prod_{j-1}$,

(ii) $j^{2} 2^{-j} \leq \operatorname{dist}\left(K_{j+1}, \Pi_{j}\right) \leq 3 j^{2} 2^{-j}$.

There are two mutually exclusive possibilities: either the sequence (4.3)

(A) terminates after $l$ steps and $\infty \in \Pi_{n+l}$, or

(B) it continues indefinitely and $\infty \notin \Pi_{j}$ for any $j$.

In the second instance there exists an infinite sequence of barriers $Q_{j}$ bounded by polygonal curves $\Gamma_{j}$ extending outward from $x_{0}$, and accumulating in a finite portion of the plane. As we shall see, this is sufficient to guarantee that $H^{2}(d \mu)$ has a BPE at $x_{0}$, a key idea in Thomson's original paper 41. An early hint at such a phenomenon can be found in Luecking [25].

Lemma 5. If there exists an infinite sequence of barriers $Q_{j}, j=n, n+1, n+2, \ldots$, surrounding a point $x_{0}$, then there is a BPE for the polynomials at $x_{0}$ in the $L^{1}(|\hat{\nu}| d A)$ norm. Hence, $H^{2}(d \mu)$ also has a BPE at $x_{0}$.

Proof. In the case of the initial barrier $Q_{n}$ it will be convenient to occasionally drop the subscript $n$ and simply write $Q=Q_{n}$ and $\Gamma=\Gamma_{n}$. Because $Q$ is a barrier, $\Gamma$ is the union of certain specified sides of $n$-th generation squares $S$ such that $\left|E_{\lambda} \cap S\right| \leq \frac{1}{100}|S|$; or, setting $F_{\lambda}=\{z:|\hat{\nu}(z)| \geq \lambda\}$, squares $S$ for which

$$
\left|F_{\lambda} \cap S\right| \geq \frac{99}{100}|S| .
$$

We can assume with no loss of generality that $\lambda=1$, and we again drop the subscript writing $F=F_{1}$ and $E=E_{1}$.

The map $L: P \rightarrow P\left(x_{0}\right)$ can be viewed as a bounded linear functional on the space of polynomials when the latter is endowed with the norm $\|P\|_{L^{\infty}(\Gamma)}=\sup _{\Gamma}|P|$. As such, $L$ can be extended in a norm-preserving way to $C(\Gamma)$, the full space of continuous functions 
on $\Gamma$ likewise endowed with the uniform norm. Hence, there exists a measure $\omega$ of finite total variation on $\Gamma$ such that $\|\omega\|=\|L\|$ and

$$
P\left(x_{0}\right)=\int_{\Gamma} P d \omega
$$

for all polynomials $P$. The first step in the proof of the lemma is to replace $\int_{\Gamma} P d \omega$ by an area integral over $F \cap Q$ committing only a small error.

Assume for the moment that $P$ is a fixed polynomial. Take $\epsilon>0$ and let $\Gamma=\bigcup I_{j}$ be the union of finitely many closed intervals $I_{j}$ with mutually disjoint interiors chosen so that

$$
\left|\int_{\Gamma} P d \omega-\sum_{j} P\left(\xi_{j}\right) \omega_{j}\right|<\epsilon
$$

whenever $\xi_{j} \in I_{j}$ and $\omega_{j}=\omega\left(I_{j}\right)$. This can be done in such a way that each $I_{j}$ is contained entirely within the side of a single square $S$ in the barrier $Q$; we need only ensure that the vertices of any square which lie on $\Gamma$ are also endpoints of some $I_{j}$. Because each point of $\Gamma=\Gamma_{n}$ is a peak point for the uniform algebra on $\Pi_{n}$ generated by the polynomials, the measure $\omega$ can have no mass concentrated at a single point. Consequently, there is no ambiguity associated with the approximating sums for $\int_{\Gamma} P d \omega$ as presented in (4.4).

Consider now a fixed barrier square $S \subseteq Q$ with one or more of its sides in $\Gamma$, let $x_{S}$ denote its center, and let $\xi_{j}$ be one of the points in (4.4) situated on $\partial S$. Since by construction $\operatorname{dist}\left(\Gamma_{n}, \Gamma_{n+1}\right) \geq n^{2} 2^{-n}$, a variant of Schwarz's lemma implies that

$$
\left|P\left(\xi_{j}\right)-P\left(x_{S}\right)\right| \leq \frac{2^{n+1}}{n^{2}}\left|\xi_{j}-x_{S}\right|\|P\|_{L^{\infty}\left(\Gamma_{n+1}\right)} \leq \frac{\sqrt{2}}{n^{2}}\|P\|_{L^{\infty}\left(\Gamma_{n+1}\right)} .
$$

Summing over only those indices $j$ for which $I_{j} \subseteq \partial S$ it follows that

$$
\left|\sum_{I_{j} \subseteq \partial S}\left(P\left(\xi_{j}\right) \omega_{j}-P\left(x_{S}\right) \omega_{j}\right)\right| \leq \frac{\sqrt{2}}{n^{2}}\|P\|_{L^{\infty}\left(\Gamma_{n+1}\right)} \sum_{I_{j} \subseteq \partial S}\left|\omega\left(I_{j}\right)\right| .
$$

Here we have passed from $\Gamma_{n}$ to $\Gamma_{n+1}$ for the sole purpose of obtaining a factor of $n^{-2}$ on the right side of (4.5). If, in addition, $B$ is the disk inscribed in $S$ with center at $x_{S}$, then

$$
P\left(x_{S}\right)=\frac{1}{|B|} \int_{B} P d A .
$$

Since $|F \cap B| \geq \frac{98}{100}|B|$ or, equivalently, $|E \cap B| \leq \frac{2}{100}|B|$ by virtue of the fact that $|F \cap S| \geq \frac{99}{100}|S|$, we have

$$
\left|P\left(x_{S}\right)-\frac{1}{|B|} \int_{F \cap B} P d A\right| \leq \frac{1}{|B|} \int_{E \cap B}|P| d A \leq \frac{2}{100}\|P\|_{L^{\infty}\left(\Gamma_{n}\right)} .
$$

Multiplying by $\omega_{j}$ and again summing over the indices for which $I_{j} \subseteq \partial S$ yields the estimate

$$
\left|\sum_{I_{j} \subseteq \partial S} P\left(x_{S}\right) \omega_{j}-\frac{\omega(S)}{|B|} \int_{F \cap B} P d A\right| \leq \frac{2}{100}\|P\|_{L^{\infty}\left(\Gamma_{n}\right)} \sum_{I_{j} \subseteq \partial S}\left|\omega\left(I_{j}\right)\right| .
$$

Thus, if $S_{1}, \ldots, S_{k}$ represent the totality of squares in $Q$ with sides along $\Gamma$ and $B_{1}, \ldots, B_{k}$ are the corresponding inscribed disks, it can be inferred from (4.4), (4.5), (4.6) and the fact that $\Gamma_{n}$ lies entirely inside the region bounded by $\Gamma_{n+1}$ that

$$
\left|P\left(x_{0}\right)-\int_{F \cap Q} P h_{n} d A\right| \leq \epsilon+\left(\frac{\sqrt{2}}{n^{2}}+\frac{2}{100}\right)\|\omega\|\|P\|_{L^{\infty}\left(\Gamma_{n+1}\right)},
$$


where $h_{n}=\sum_{j=1}^{k} \frac{\omega\left(S_{j}\right)}{\left|B_{j}\right|} \chi_{F \cap B_{j}}$. Hence, $h_{n}=\sum_{j=1}^{k} \frac{4}{\pi} 2^{2 n} \omega\left(S_{j}\right) \chi_{F \cap B_{j}}$ since all squares $S_{j}$ are from the $n$-th generation. Since $\epsilon>0$ is arbitrary it can now be dropped from the inequality, and by choosing $n$ sufficiently large for the initial barrier $Q=Q_{n}$ of the presumed sequence we can arrange that

$$
\left|P\left(x_{0}\right)-\int_{F \cap Q_{n}} P h_{n} d A\right| \leq \frac{3}{100}\|\omega\|\|P\|_{L^{\infty}\left(\Gamma_{n+1}\right)}
$$

for all polynomials $P$, and $\left\|h_{n}\right\|_{\infty} \leq \frac{2^{2 n+2}}{\pi}\|\omega\|$.

The entire process can now be repeated. In view of (4.7) the map

$$
L_{n+1}: P \rightarrow P\left(x_{0}\right)-\int_{F \cap Q_{n}} P h_{n} d A
$$

can be extended from the space of polynomials and treated as a bounded linear functional on $C\left(\Gamma_{n+1}\right)$ with $\left\|L_{n+1}\right\| \leq \frac{3}{100}\|L\|$, where $L=L_{n}$ and where $\|L\|=\|\omega\|$. Since all squares adjacent to $\Gamma_{n+1}$ are barrier squares from $Q_{n+1}$, the argument presented above allows us to conclude that there exists a function $h_{n+1}$ with support in $F \cap Q_{n+1}$ such that

$$
\left|P\left(x_{0}\right)-\int P h_{n} d A-\int P h_{n+1} d A\right| \leq\left(\frac{3}{100}\right)^{2}\|L\|\|P\|_{L^{\infty}\left(\Gamma_{n+2}\right)}
$$

for all polynomials $P$, and $\left\|h_{n+1}\right\|_{\infty} \leq \frac{4}{\pi} 2^{2(n+1)}\left(\frac{3}{100}\right)\|L\|$. Continuing in this way we obtain an infinite sequence of functions $h_{n}, h_{n+1}, \ldots$ such that for any $k>0$,

$$
\left|P\left(x_{0}\right)-\int P\left(h_{n}+\cdots+h_{n+k}\right) d A\right| \leq\left(\frac{3}{100}\right)^{k+1}\|L\|\|P\|_{L^{\infty}\left(\Gamma_{n+k+1}\right)} .
$$

Moreover, for a given polynomial $P$ the right side tends to zero as $k \rightarrow+\infty$, since the curves $\Gamma_{n+k}$ all lie in a bounded portion of the plane. Therefore, setting $h=\sum_{k=0}^{\infty} h_{n+k}$ it follows that $h$ is supported on $F=\{z:|\hat{\nu}(z)| \geq 1\}$ and

$$
P\left(x_{0}\right)=\int P h d A
$$

for all polynomials $P$. Also, $h \in L^{\infty}$ because the individual $h_{j}$ 's have disjoint supports and $\left\|h_{n+k}\right\|_{\infty} \leq\left\|h_{n}\right\|_{\infty}$ for all $k>0$; hence, $\|h\|_{\infty}=\left\|h_{n}\right\|_{\infty}$. Inasmuch as $h$ is supported on the set where $|\hat{\nu}| \geq 1$, it follows that

$$
\left|P\left(x_{0}\right)\right| \leq\|h\|_{\infty} \int|P||\hat{\nu}| d A,
$$

from which we conclude that there is a BPE for the polynomials at $x_{0}$ in the $L^{1}(|\hat{\nu}| d A)$ norm, and so also in the $L^{2}(d \mu)$-norm.

The main theorem on BPEs can now be established along lines similar to those presented earlier under conditions which allowed the use of purely potential-theoretic methods.

Theorem 1. If $\mu$ is a positive measure of compact support in $\mathbb{C}$, not concentrated at a single point, then $H^{2}(d \mu)=L^{2}(d \mu)$ if and only if $H^{2}(d \mu)$ has no BPEs.

Proof. Suppose that $H^{2}(d \mu)$ has no BPEs. Let $g$ be any function in $L^{2}(d \mu)$ with the property that $\int P g d \mu=0$ for all polynomials $P$, and set $\nu=g \mu$. Fix a point $x_{0}$ at which the potential $U^{|\nu|}\left(x_{0}\right)<\infty$.

For an arbitrary, but fixed, $\lambda>0$ consider once again the set $E_{\lambda}=\{z:|\hat{\nu}(z)|<\lambda\}$. By Lemma 4 there are no BPEs in the $L^{1}(|\hat{\nu}| d A)$-norm, and consequently by Lemma 5 
there can be no infinite sequence of barriers associated with $\mathbb{C} \backslash E_{\lambda}$, for any $\lambda$. Beginning therefore with any generation, the $n$-th say, there exists a finite sequence of polygons

$$
\Pi_{n} \subseteq \Pi_{n+1} \subseteq \cdots \subseteq \Pi_{n+l}
$$

constructed in the manner of (4.3) with $x_{0} \in \Pi_{n}$ and $\infty \in \Pi_{n+l}$. In particular, for $n<j<n+l$ there exist compact sets $K_{j} \subseteq \Pi_{j} \backslash \Pi_{j-1}$, some of which may be empty, such that if $K_{j} \neq \varnothing$,

(i) $K_{j}$ is the union of squares in $\mathcal{G}_{j}$ and connects $\Gamma_{j-1}^{*}$ to $Q_{j}$,

(ii) $\left|E_{\lambda} \cap S\right|>\frac{1}{100}|S|$ for each square $S \subseteq K_{j}$,

(iii) $\operatorname{dist}\left(K_{j}, \Gamma_{j}^{*}\right) \leq \operatorname{dist}\left(K_{j}, \Gamma_{j}\right)+\operatorname{dist}\left(\Gamma_{j}, \Gamma_{j}^{*}\right)<2^{-j}+3 j^{2} 2^{-j}<4 j^{2} 2^{-j}$.

By assumption $K_{n+l}$ extends to $\infty$. Here the notation is consistent with that established in the discussion preceding Lemma 5 .

As in the earlier description, let $S^{*}$ be an $n$-th generation square containing $x_{0}$, and now form a chain of squares and rectangles leading from $S^{*}$ to $\infty$ as follows. Join $S^{*}$ to the first nonempty $K_{j}$ by a narrow rectangle $R_{0}$ so that $\operatorname{diam}\left(R_{0}\right) \approx \operatorname{dist}\left(S^{*}, \Gamma_{j-1}^{*}\right)$. Choose a connected component of $K_{j}$ meeting $R_{0}$, and thereby obtain a chain from $S^{*}$ to the barrier $Q_{j}$. According to property (iii) that chain can then be joined to $\Gamma_{j}^{*}$ by another narrow rectangle $R_{j}$, this time with $\operatorname{diam}\left(R_{j}\right)<4 j^{2} 2^{-j}$. At this point the resulting chain either meets $K_{j+1}$ at $\Gamma_{j}^{*}$, or it does not. If it does we adjoin a connected component of $K_{j+1}$ extending the chain to the next barrier $Q_{j+1}$; if not, we adjoin a narrow rectangle $R_{j+1}$ extending the chain to $\Gamma_{j+1}^{*}$, taking care to ensure that $\operatorname{diam}\left(R_{j+1}\right)<4(j+1)^{2} 2^{-(j+1)}$. We continue in this way until the chain of squares and rectangles from $S^{*}$ escapes to $\infty$. The result is a connected set $X$ joining $S^{*}$ to $\infty$, which is composed of squares satisfying property (ii) and certain narrow rectangles.

Given $r>0$, let $B_{r}=B\left(x_{0}, r\right)$ be the disk with center at $x_{0}$ and radius $r$. Fix $n$ large; how large depends on $r$ and will be determined presently. Choose $S^{*} \in \mathcal{G}_{n}$ with $x_{0} \in S^{*}$ and let $X$ be formed as above. By discarding certain superfluous pieces we can assume that $X \cap B_{r}$ is connected and joins $S^{*}$ to $\partial B_{r}$. Hence,

$$
\gamma\left(X \cap B_{r}\right) \geq \frac{1}{4} \operatorname{diam}\left(X \cap B_{r}\right) \geq \frac{r}{8} .
$$

Denote by $K$ the collection of all squares in $X \cap B_{r}$ that lie entirely inside $B_{r}$. By construction $K=\bigcup K_{j}$, where each $K_{j}$ is the union of squares from $\mathcal{G}_{j}$ that satisfy

(1) $\left|E_{\lambda} \cap S\right|>\frac{1}{100}|S|$ for each square $S \subseteq K$,

(2) $j^{2} 2^{-j} \leq \operatorname{dist}\left(K_{j}, \Gamma_{j}^{*}\right)<4 j^{2} 2^{-j}$.

It follows from the preceding remarks and the semiadditivity of analytic capacity that

$$
\frac{r}{16} \leq \gamma\left(X \cap B_{r / 2}\right) \leq C\left[\gamma(K)+\sum_{j=n}^{\infty} j^{2} 2^{-j}\right],
$$

where $C$ is an absolute constant. Since, by assumption, construction of the set $X$ can begin with an arbitrary generation we are free to choose $n$ as large as we please, and (4.8) remains valid with the same constant $C$. In particular, $n$ can be chosen large enough so that the infinite sum on the right side of (4.8) is negligible, from which we conclude that $\gamma(K) \geq C r$ for another absolute constant. Inasmuch as properties (1) and (2) are satisfied, Lemma 3 implies that

$$
\gamma\left(E_{\lambda} \cap B_{r}\right) \geq \tilde{\gamma}\left(E_{\lambda} \cap B_{r}\right) \geq C \epsilon \gamma(K) \geq C \epsilon r,
$$

for some constant $\epsilon$ independent of $r$, and therefore $E_{\lambda}$ is thick at $x_{0}$ in the sense that

$$
\limsup _{r \rightarrow 0} \frac{\gamma\left(E_{\lambda} \cap B_{r}\right)}{r}>0 \text {. }
$$


For a given $r$, once $n$ has been fixed the set $K$ consists of finitely many squares for which (1) is satisfied. Since $U^{|\nu|}<\infty$ a.e.- $d A$, we can find a subset $\Omega_{r} \subseteq\left(E_{\lambda} \cap B_{r}\right)$ on which $U^{|\nu|}$ is bounded and for which property (1) is preserved, that is, for which $\left|\Omega_{r} \cap S\right|>\frac{1}{100}|S|$ for every square $S \subseteq K$. By the same reasoning presented above in support of the lower estimate for $\gamma\left(E_{\lambda} \cap B_{r}\right)$ it follows that $\gamma\left(\Omega_{r}\right) \geq C \epsilon r$, and since $\gamma\left(E_{\lambda} \cap B_{r}\right) \leq r$, that

$$
\gamma\left(\Omega_{r}\right) \geq C \epsilon \gamma\left(E_{\lambda} \cap B_{r}\right) .
$$

The hypotheses of Lemma 2 are therefore satisfied, and so

$$
\left|\hat{\nu}\left(x_{0}\right)\right| \leq \limsup _{\substack{z \rightarrow x_{0} \\ z \in E_{\lambda}}}|\hat{\nu}(z)| \leq \lambda .
$$

Since the inequality is valid for all $\lambda>0$, we can infer that $\hat{\nu}\left(x_{0}\right)=0$. Hence, $\hat{\nu}=0$ a.e.- $d A$, and $\nu=g \mu=0$ as a measure. Therefore, $H^{2}(d \mu)=L^{2}(d \mu)$.

\section{§5. Some ADditional COROLlaries}

The bounded point evaluation question, which is the principal focus of this investigation, is a special case of a more general problem, various aspects of which have been extensively examined over many years: Given a subset $X \subseteq \mathbb{C}$, a Banach space $B$ of functions defined on $X$ and a subfamily $\mathcal{F} \subset B$ of functions analytic in a neighborhood of $X$, is $\mathcal{F}$ dense in $B$ ? The difficulties can be quite varied depending on the choice of $X$, the space $B$ and the family $\mathcal{F}$. However, it often happens that either $\mathcal{F}$ is dense in $B$ or the only functions that can be so approximated have a natural analytic extension outside of $X$. This is especially true in case $\mathcal{F}$ is the set of all complex analytic polynomials (cf. [6]).

Illustrations of the continuation phenomenon associated with polynomial approximation occur in the following familiar settings, when

(1) $X$ is compact and $B=A(X)$ is the space of all functions continuous on $X$, analytic in its interior, and endowed with the uniform norm;

(2) $X=\Omega$ is a simply connected domain and $B=L_{a}^{p}(\Omega, d A), 1 \leq p<\infty$, is the set of all functions in $L^{p}(\Omega, d A)$ that are analytic in $\Omega$.

In the case of uniform approximation suppose, for example, that $X$ is a compact set which separates the plane, so that $\mathbb{C} \backslash X$ has at least one bounded component $G$. Then any sequence of polynomials that converges uniformly on $X$ necessarily converges uniformly on $X \cup G$ as well. This is an obstacle to uniform polynomial approximation in $A(X)$, since the limit of any such sequence admits an analytic continuation to $G$. In particular, if $a \in G$, then $(z-a)^{-1} \in A(X)$, but $(z-a)^{-1}$ cannot be uniformly approximated on $X$ by polynomials. On the other hand, by a theorem of Mergelyan (cf. [14, p. 48]) this is the only obstacle to the density of the polynomials in $A(X)$. In other words, the density-continuation dichotomy is valid here for a purely topological reason.

While the polynomial approximation question for $A(X)$ can be settled in this way, the same cannot be said for approximation in the $L^{p}(\Omega, d A)$-norm. That discovery was made by Keldysh in 1939 (cf. [35, p. 117]). If $\Omega$ is a crescent domain, that is, a domain topologically equivalent to the region bounded by two internally tangent circles, he showed that the polynomials may or may not be dense in $L_{a}^{p}(\Omega, d A)$ depending on the thickness of $\Omega$ near the multiple boundary point. Again, however, if density fails, then the only functions in $L_{a}^{p}(\Omega, d A)$ which admit approximation by polynomials can be continued analytically across $\partial \Omega$ into the bounded component of $\mathbb{C} \backslash \bar{\Omega}$, another instance of the density-continuation dichotomy.

In 1955 Mergelyan 36 conjectured that this is the only way that the polynomials can fail to be dense in $L_{a}^{p}(\Omega, d A)$ for any bounded simply connected domain $\Omega$. Subsequently, 
the author [5], using potential-theoretic ideas introduced by Hedberg [21] and Maz'ya and Havin [28, 29] to study approximation in the mean by analytic functions, verified the conjecture in the more general setting of approximation in $L_{a}^{p}(\Omega, w d A)$ for a weighted measure $w d A$ with $w \in L^{1+\epsilon}(d A) \cap C(\Omega)$. The same phenomenon can be observed in connection with the Bernstein problem for weighted polynomial approximation on the entire real line, in both the weighted uniform and weighted $L^{p}$ metrics (cf. [6). The following was noted by Thomson [41] and is further evidence of the reciprocal relation between approximation and analytic continuation.

Corollary 1. Let $\mu$ be a positive measure of compact support in the complex plane $\mathbb{C}$. Then, either $H^{2}(d \mu)=L^{2}(d \mu)$ or there exists a point $x_{0}$ and an open set $U$ containing $x_{0}$ such that every $f \in H^{2}(d \mu)$ admits an analytic continuation to $U$.

The corollary is actually valid for approximation in $L^{p}(d \mu), 1 \leq p<\infty$, and the proof presented here can easily be adapted to cover the added situations.

Proof. Suppose that $H^{2}(d \mu) \neq L^{2}(d \mu)$. Then $H^{2}(d \mu)$ has a BPE at some point $x_{0}$, and so there exists $g \in L^{2}(d \mu)$ with

$$
P\left(x_{0}\right)=\int P g d \mu
$$

for all polynomials $P$. Hence, $\nu=\left(z-x_{0}\right) g \mu$ is an annihilating measure for which

(i) $\hat{\nu}\left(x_{0}\right)=1$,

(ii) $U^{|\nu|}\left(x_{0}\right)=\int|g| d \mu<\infty$.

For some $\lambda>0$, according to the proof of Theorem 1, the set $F_{\lambda}=\{z:|\hat{\nu}(z)| \geq \lambda\}$ gives rise to an infinite sequence of barriers $Q_{j}, j=n, n+1, \ldots$, surrounding $x_{0}$ and extending outward from there such that

$$
\left|F_{\lambda} \cap S\right| \geq \frac{99}{100}|S|
$$

for all barrier squares $S$ in each generation $j=n, n+1, \ldots$. Repeating the argument in the proof of Lemma 5 , it follows that each point $\xi$ in the region bounded by the initial barrier $Q_{n}$ corresponds to a BPE with norm depending only on $\operatorname{dist}\left(\xi, Q_{n}\right)$. Thus, if $U$ is a neighborhood of $x_{0}$ with $\operatorname{dist}\left(U, Q_{n}\right)>0$ there exists a fixed constant $C>0$ such that

$$
|P(\xi)| \leq C\|P\|_{L^{2}(d \mu)}
$$

for all $\xi \in U$ and all polynomials $P$. Therefore, every $f \in H^{2}(d \mu)$ admits an analytic continuation to $U$.

Added in translation. To allow for the possibility that $\mu$ may contain point masses, let $\nu=g \mu$ be any nonzero annihilator of $H^{2}(\mu)$. Since $|\hat{\nu}|>0$ on a set of positive $d A$ measure we can choose a point $x_{0}$ where $\hat{\nu}\left(x_{0}\right) \neq 0$ and $U^{|\nu|}\left(x_{0}\right)<\infty$, and the proof proceeds as before.

The following two well-known results of Lavrentiev and Vitushkin are also immediate consequences of the approximation scheme outlined above and, in particular, consequences of Lemma 2. Given a compact set $X \subset \mathbb{C}$ let $P(X)$ and $R(X)$ denote the closures in $C(X)$ of the polynomials and rational functions, respectively.

Corollary 2 (Lavrentiev). $P(X)=C(X)$ if and only if $X$ has no interior and the complement $\mathbb{C} \backslash X$ is connected. 
Proof. Let $\nu$ be any measure on $X$ with the property that $\int P d \nu=0$ for all polynomials $P$, and form the Cauchy transform $\hat{\nu}$. Since $\hat{\nu}$ is analytic in the connected region $\mathbb{C} \backslash X$ and $\hat{\nu}=0$ in a neighborhood of $\infty$, it follows that $\hat{\nu} \equiv 0$ in $\mathbb{C} \backslash X$. If $|X|=0$, then $\hat{\nu}=0$ a.e.- $d A$, from which we can infer that $\nu=0$ as a measure. Hence, $P(X)=C(X)$.

We may assume, therefore, that $|X|>0$. Fix a point $x_{0} \in X$ where $U^{|\nu|}\left(x_{0}\right)<\infty$ and let $B_{r}=B\left(x_{0}, r\right)$ be the disk of radius $r$ with center at $x_{0}$. Because $\mathbb{C} \backslash X$ is connected we can find a compact set $E_{r} \subset B_{r} \backslash X$, an arc for example, with diam $E_{r} \geq \frac{r}{2}$, and so $\gamma\left(E_{r}\right) \geq \frac{r}{8}$. Since $U^{|\nu|}$ is bounded on $E_{r}$ and

$$
\limsup _{r \rightarrow 0} \frac{\gamma\left(B_{r} \backslash X\right)}{r}>0,
$$

the requirements of Lemma 2 are satisfied and

$$
\left|\hat{\nu}\left(x_{0}\right)\right| \leq \limsup _{\substack{z \rightarrow x_{0} \\ z \in B_{r} \backslash X}}|\hat{\nu}(z)|=0 .
$$

Inasmuch as $U^{|\nu|}<\infty$ a.e.- $d A$ on $X$, if follows that $\hat{\nu}=0$ a.e.- $d A$. Again,

$$
P(X)=C(X) .
$$

Corollary 3 (Vitushkin). $R(X)=C(X)$ if and only if

$$
\limsup _{r \rightarrow 0} \frac{\gamma(B(x, r) \backslash X)}{r}>0
$$

for $d A$ almost every $x \in X$.

Proof. Let $\nu$ be a measure on $X$ such that $\int f d \nu=0$ for every rational function $f$ with poles off $X$. Clearly, $\hat{\nu} \equiv 0$ in $\mathbb{C} \backslash X$ and just as in Corollary 2 the capacitary density assumption implies that $\hat{\nu}=0$ a.e.- $d A$ on $X$. Hence, $R(X)=C(X)$. For a proof in the other direction see [14, p. 207].

Acknowledgement. I am grateful to the Institut Mittag-Leffler and to the Centre De Recerca Matematica of the Institut D'Estudis Catalans for providing support and a comfortable work environment during my sabbatical leave. I especially want to thank Joan Verdera for calling my attention to the then unpublished work of Xavier Tolsa, and John Wermer through whose influence I was initially drawn to this and an entire circle of related problems which have held my interest over many years.

\section{REFERENCES}

[1] D. R. Adams and L. I. Hedberg, Function spaces and potential theory, Grundlehren Math. Wiss., vol. 314, Springer-Verlag, Berlin, 1996. MR 1411441 (97j:46024)

[2] L. V. Ahlfors, Bounded analytic functions, Duke Math. J. 14 (1947), 1-11. MR.0021108 (9:24a)

[3] J. Bram, Subnormal operators, Duke Math. J. 22 (1955), 75-94. MR0068129 (16:835a)

[4] J. E. Brennan, Invariant subspaces and weighted polynomial approximation, Ark. Mat. 11 (1973), 167-189. MR0350398 (50:2891)

[5] _ Point evaluations, invariant subspaces and approximation in the mean by polynomials, J. Funct. Anal. 34 (1979), 407-420. MR0556263 (81h:46026)

[6] , The Cauchy integral and analytic continuation, Math. Proc. Cambridge Philos. Soc. 97 (1985), 491-498. MR0778684 (86d:30010)

[7] S. W. Brown, Some invariant subspaces for subnormal operators, Integral Equations Operator Theory 1 (1978), 310-333. MR0511974(80c:47007)

[8] L. Carleson, Mergelyan's theorem on uniform polynomial approximation, Math. Scand. 15 (1964), 167-175. MR0198209 (33:6368)

[9] L Lars Ahlfors and the Painlevé problem, In the Tradition of Ahlfors and Bers (Stony Brook, NY, 1998), Contemp. Math., vol. 256, Amer. Math. Soc., Providence, RI, 2000, pp. 5-10. MR1759666 (2001e:30037) 
[10] J. B. Conway, The theory of subnormal operators, Math. Surveys Monogr., vol. 36, Amer. Math. Soc., Providence, RI, 1991. MR1112128 (92h:47026)

[11] A. M. Davie, Analytic capacity and approximation problems, Trans. Amer. Math. Soc. 171 (1972), 409-444. MR0350009 (50:2502)

[12] J. Deny and J. L. Lions, Les espaces du type de Beppo Levi, Ann. Inst. Fourier (Grenoble) 5 (1953-1954), 305-370 (1955). MR0074787(17:646a)

[13] W. H. Fleming, Functions whose partial derivatives are measures, Illinois J. Math. 4 (1960), 452478. MR0130338 (24:A202)

[14] T. W. Gamelin, Uniform algebras, Prentice-Hall, Englewood Cliffs, NJ, 1969. MR0410387 $(53: 14137)$

[15] J. B. Garnett, Positive length but zero analytic capacity, Proc. Amer. Math. Soc. 24 (1970), 696699. MR0276456 (43:2203)

[16] _ Analytic capacity and measure, Lecture Notes in Math., vol. 297, Springer-Verlag, BerlinNew York, 1972. MR0454006 (56:12257)

[17] P. R. Halmos, Normal dilations and extensions of operators, Summa Brasil. Math. 2 (1950), 125134. MR0044036 (13:359b)

[18] F. Hartogs and A. Rosenthal, Über Folgen analytischer Funktionen, Math. Ann. 104 (1931), 606610.

[19] S. Ya. Khavinson, Representation and approximation of functions on thin sets, Contemporary Problems in Theory Anal. Functions (Internat. Conf., Erevan, 1965), "Nauka", Moscow, 1966, pp. 314-318. (Russian) MR0210923 (35:1808)

[20] _ Golubev sums: a theory of extremal problems like the analytic capacity problem and of related approximation processes, Uspekhi Mat. Nauk 54 (1999), no. 4, 75-142; English transl., Russian Math. Surveys 54 (1999), no. 4, 753-818. MR.1741279(2001b:30041)

[21] L. I. Hedberg, Non-linear potentials and approximation in the mean by analytic functions, Math. Z. 129 (1972), 299-319. MR0328088 (48:6430)

[22] L. D. Ivanov, Variations of sets and functions, "Nauka", Moscow, 1975. (Russian) MR0476953 $(57: 16498)$

[23] S. V. Khrushchev, The Brennan alternative for measures with finite entropy, Izv. Akad. Nauk Armyan. SSR Ser. Mat. 14 (1979), no. 3, 184-191; English transl., Soviet J. Contemp. Math. Anal. 14 (1979), 27-33. MR0553495 (81a:46032)

[24] N. S. Landkof, Foundations of modern potential theory, Grundlehren Math. Wiss., vol. 180, Springer-Verlag, New York-Heidelberg, 1972. MR0350027 (50:2520)

[25] D. H. Luecking, Inequalities on Bergman spaces, Illinois J. Math. 25 (1981), 1-11. MR0602889 (82e:30072)

[26] J. Mateu, X. Tolsa, and J. Verdera, On the semiadditivity of analytic capacity and planar Cantor sets, Harmonic Analysis at Mount Holyoke (South Hadley, MA, 2001), Contemp. Math., vol. 320, Amer. Math. Soc., Providence, RI, 2003, pp. 259-278. MR1979945 (2005f:30050)

[27] P. Mattila, M. S. Mel'nikov, and J. Verdera, The Cauchy integral, analytic capacity, and uniform rectifiability, Ann. of Math. (2) 144 (1996), 127-136. MR1405945 (97k:31004)

[28] V. G. Maz'ya and V. P. Havin, A nonlinear potential theory, Uspekhi Mat. Nauk 27 (1972), no. 6, 67-138; English transl., Russian Math. Surveys 27 (1972), no. 6, 71-148. MR0409858 (53:13610)

[29] - Applications of $(p, l)$-capacity to some problems in the theory of exceptional sets, Mat. Sb. 90 (1973), no. 4, 558-591; English transl., Math. USSR-Sb. 19 (1973), 547-580. MR0367207 $(51: 3449)$

[30] M. S. Mel'nikov, On the Gleason parts of the algebra $R(X)$, Mat. Sb. 101 (1976), no. 2, 293-300; English transl., Math. USSR-Sb. 30 (1976), 261-268. MR0425619(54:13573)

[31] _ Analytic capacity: discrete approach and the curvature of measure, Mat. Sb. 186 (1995), no. 6, 57-76; English transl., Sb. Math. 186 (1995), no. 6, 827-846. MR1349014 (96f:30020)

[32] _ The saga of the Painlevé problem and of analytic capacity, Trudy Mat. Inst. Steklov. 235 (2001), 157-164; English transl., Proc. Steklov Inst. Math. 235 (2001), no. 4, 150-157. MR1886580 (2003a:30001)

[33] M. S. Mel'nikov and S. O. Sinanyan, Questions in the theory of approximation of functions of one complex variable, Itogi Nauki i Tekhniki, Sovrem. Probl. Mat., vol. 4, VINITI, Moscow, 1975, pp. 143-250; English transl., J. Soviet Math. 5 (1976), 688-752. MR0499179 (58:17109)

[34] M. S. Mel'nikov and J. Verdera, A geometric proof of the $L^{2}$-boundedness of the Cauchy integral on Lipschitz graphs, Internat. Math. Res. Notices 1995, no. 7, 325-331. MR1350687 (96f:45011)

[35] S. N. Mergelyan, On completeness of systems of analytic functions, Uspekhi Mat. Nauk (N.S.) 8 (1953), no. 4, 3-63; English transl., Amer. Math. Soc. Transl. Ser. 219 (1962), 109-166. MR0058698 $(15: 411 \mathrm{f})$ 
[36] General metric criteria for the completeness of systems of polynomials, Dokl. Akad. Nauk SSSR 105 (1955), no. 5, 901-904. (Russian) MR0075336(17:730b)

[37] N. G. Meyers, A theory of capacities for potentials of functions in Lebesgue classes, Math. Scand. 26 (1970), 255-292 (1971). MR0277741 (43:3474)

[38] H. Pajot, Analytic capacity, rectifiability, Menger curvature and Cauchy integral, Lecture Notes in Math., vol. 1799, Springer-Verlag, Berlin, 2002. MR1952175 (2004d:28009)

[39] E. M. Stein, Singular integrals and differentiability properties of functions, Princeton Math. Series, No. 30, Princeton Univ. Press, Princeton, NJ, 1970. MR0290095 (44:7280)

[40] J. E. Thomson, Invariant subspaces for algebras of subnormal operators, Proc. Amer. Math. Soc. 96 (1986), 462-464. MR0822440 (87i:47005)

[41] _ Approximation in the mean by polynomials, Ann. of Math. (2) 133 (1991), 477-507. MR 1109351 (93g:47026)

[42] X. Tolsa, $L^{2}$-boundedness of the Cauchy integral operator for continuous measures, Duke Math. J. 98 (1999), 269-304. MR.1695200 (2000d:31001)

[43] (2003m:31004)

[44] _ Painlevé's problem and the semiadditivity of analytic capacity, Acta Math. 190 (2003), 105-149. MR 1982794 (2005c:30020)

[45] J. Verdera, $L^{2}$-boundedness of the Cauchy integral and Menger curvature, Harmonic Analysis and Boundary Value Problems (Fayetteville, AR, 2000), Contemp. Math., vol. 277, Amer. Math. Soc., Providence, RI, 2001, pp. 139-158. MR1840432 (2002f:30052)

[46] A. G. Vitushkin, An example of a set of positive length, but of zero analytic capacity, Dokl. Akad. Nauk SSSR 127 (1959), no. 2, 246-249. (Russian) MR0118838(22:9607)

[47] _ Analytic capacity of sets and problems in approximation theory, Uspekhi Mat. Nauk 22 (1967), 141-199; English transl., Russian Math. Surveys 22 (1967), 139-200. MR0229838|(37:5404)

[48] J. Wermer, Report on subnormal operators, Report of an International Conference on Operator Theory and Group Representations (Arden House, Harriman, NY, 1955), Publ. 387, Nat. Acad. Sci.-Nat. Res. Council, Washington, DC, 1955, pp. 1-3. MR0076316 (17:880c)

[49] L. Zalcman, Analytic capacity and rational approximation, Lecture Notes in Math., vol. 50, Springer-Verlag, Berlin-New York, 1968. MR0227434 (37:3018)

[50] W. P. Ziemer, Extremal length as a capacity, Michigan Math. J. 17 (1970), 117-128. MR0268401 (42:3299)

Department of Mathematics, University of Kentucky, Lexington, Kentucky 40506

E-mail address: brennan@ms.uky.edu

Received 24/MAY/2004

Translated by THE AUTHOR 